\title{
SENSORY PROFILE IN THE SNOEZELEN INTERVENTION
}

\author{
María José Cid Rodriguez \\ Ramona Ribes Castells, Kateřina Janků
}

\begin{abstract}
In this article we present a tool for professionals who work in care services (24-hour services) with people with a type of dependency and/or developmental disability, such as dementia and/or Alzheimer's, intellectual disabilities, autism spectrum disorders etc. Sensory interventions for these people are primarily focused on offering them better quality of life and increased emotional well-being.

Sensations are the basis of well-being and are present and functioning in everyone, regardless of their level of dependence. The main challenge for professionals and institutions is to provide the most appropriate intervention to each person and, thereby, ensure a pleasant and enjoyable environment and daily activities that accord with his/her capabilities. It is necessary to start from the most reliable knowledge possible of the sensory capacities, tastes, and sensory preferences of the subject.

The article includes practical examples of Sensory Assessments that allow us to elaborate the Sensory Profile of a person, involving every sense (visual, auditory, tactile, proprioceptive, olfactory, gustatory, and vestibular), in order to identify the sensory capacities of the person, his/her tastes, and sensations of displeasure and discomfort. Based on qualitative observational methods, the article provides a practical guide to performing a Sensory Assessment.
\end{abstract}

\section{Keywords}

Snoezelen, sensory profile, sensory assessment, sensations, sensory intervention

\section{Introduction}

Our emotional well-being begins with our ability to have daily experiences appropriate to our basic neurological capacities, i.e., our sensations. From the developmental 
and learning pyramid of Williams \& Shellenberg (1996), we know that all the information that reaches us stems from our basic sensations.

\section{Figure 1 Development and learning Pyramid. (Williams \& Shellenberger, 1996)}

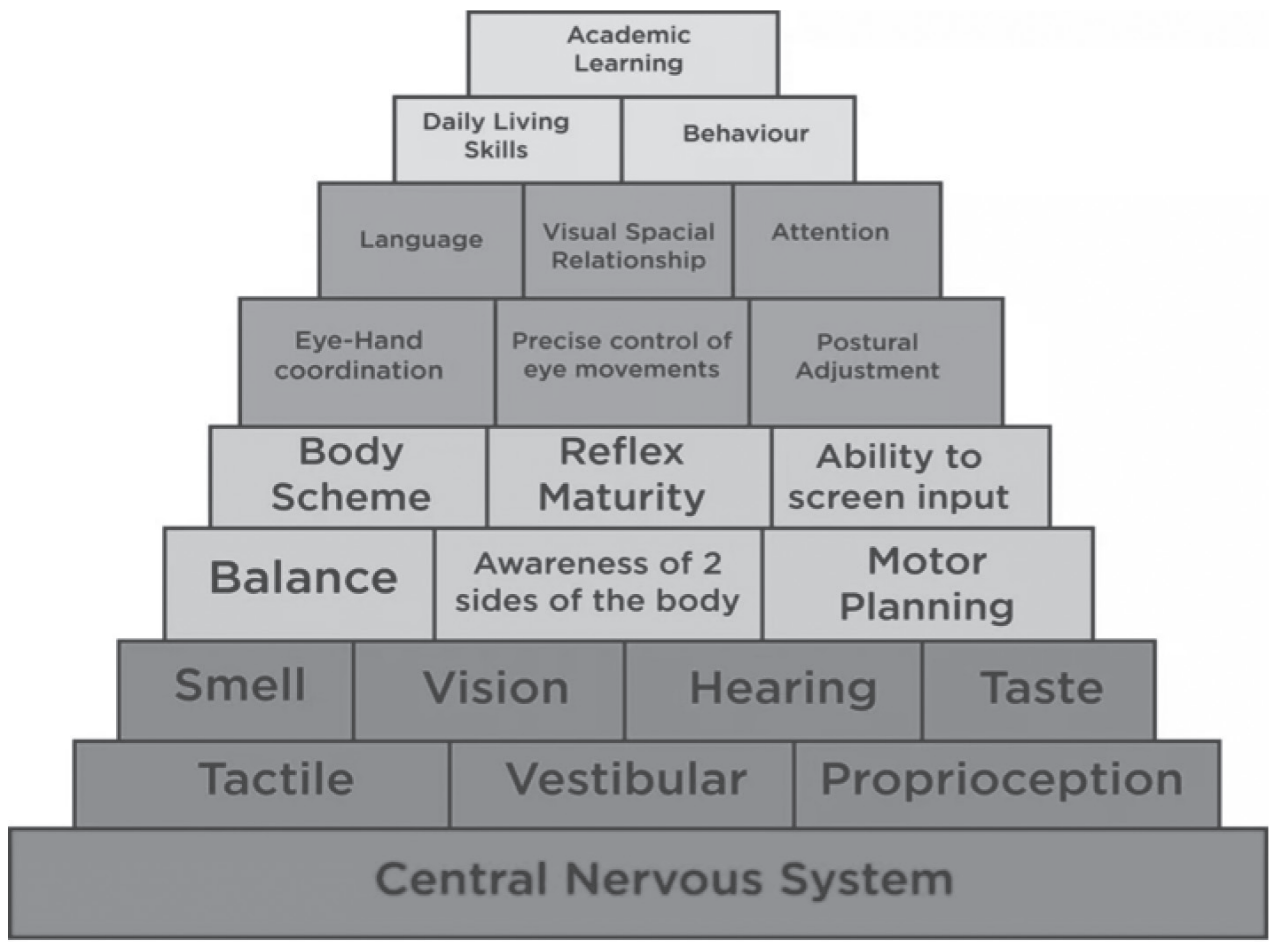

The first thing we must ask ourselves is what are the basic sensations? According to the developmental pyramid above, these are the sense of touch, the vestibular (or kinesthetic) sense, and the proprioceptive sense. We call them basic sensations because they are the most ancient and primitive sensations. The first sensations that developed in our phylogenetic ancestors were these basic sensations. Also, at the ontogenetic level, they are the first to start operating. In ontogenetic evolution, when the fetus begins to form in the mother's womb, the first sensory capacities that appear in the amniotic fluid are the sensations of touch, vestibularity, and proprioception. These three basic sensations are also the last to disappear.

At the end of life, the Williams and Shellenberg developmental pyramid operates in reverse. As advanced deterioration occurs, cognitive and executive abilities may disappear, due to possible neurological changes to the prefrontal lobe. However, the basic sensations, located in the lower parts of the cortex, are better preserved. It should also be noted that these sensations are close to the limbic system, and therefore, to the emotionality of an individual. For this reason, it is important to understand that while a person with 
advanced cognitive impairment may have compromised cognitive abilities, they retain the ability to feel emotions, primarily linked to the most basic sensations.

Above the basic sensations, we find the sensations of Sight, Hearing, Smell and Taste. These sensations are essential for the daily functioning of the individual. The correct functioning of the different sensory systems will allow a person to be connected with his/ her environment and with him/herself in a satisfactory manner.

For each individual, especially those with a type of neurological disorder, developmental delay, intellectual disability, mental illness, autism, etc., the functioning of their various sensory systems is essential for their well-being. A person will have particular sensory capacities, determined by different factors: their genetics, their habits, their environment, their cultural context, their condition on a given day, and, above all, the presence or absence of a physical, neurological, or other type of disorder.

The Snoezelen intervention makes it possible to provide the person with emotional wellbeing through their sensations. The Snoezelen philosophy in this sense is predicated on generating sensory experiences for the person that create a pleasant day-to-day existence, through appropriate and varied sensory experiences. Sensoriality is important in all people, but it is of particular importance in the profiles of people such as those mentioned above (people with cognitive impairment, dementia, Alzheimer's, intellectual disability, mental illness, autism, etc). The Snoezelen intervention should, therefore, allow us to provide sensory experiences in the most suitable way possible to support the physical and emotional well-being of the person.

In order to plan an appropriate sensory intervention, it is first necessary to be aware of the sensory capacities of an individual: to obtain the most reliable information possible on each of their senses and their functioning. In addition to his/her sensory capabilities, it is important to know the sensory preferences of each person: to know which intensities and characteristics of each stimulus generate well-being or, on the contrary, cause discomfort or displeasure. In this way, we can establish a baseline, or starting point which we can use to plan a precise personalised sensory intervention. All such sensory information about the person, his/her sensory capacities, his/her preferences, or his/her sensory sensations of displeasure make up what we call their Sensory Profile.

The benchmark in terms of Sensory Profile and Sensory History is the work of Winnie Dunn and her collaborators. Dunn's Sensory Profile (Dunn, 1994, Tomchek, Dunn, 2007), in its original version, is divided into six sensory categories (auditory, visual, taste/smell, movement, body position, and touch) and two behavioural categories (emotional/ social, and activity level), with a total of 125 items. A principal component factor analysis of the Sensory Profile was carried out based on the responses of 1,115 children aged 3 to 10 years who had typical development (Dunn and Westman, 1997, Brown et al, 2001). The resulting factor structure did not suggest categories of sensory modalities but rather patterns of behavioural response. This factor analysis led to the development of Dunn's (1997) Sensory Processing Model, which characterizes four different sensory processing tendencies (people with low sensory registration, sensation seekers, sensory sensitive people, and sensation avoidants). 
One way to measure sensory processing from a behavioural perspective is to obtain a sensory history. Advantages of sensory histories over other measures include ease of administration and contextual relevance (Dunn, 1994). Sensory histories are contextually relevant because they allow the assessment of behaviours in the natural environment rather than the commonly used alternative of performance observation in a clinical setting. In addition, sensory histories allow the person or family member who is the focus of the history taking to be an active participant in the assessment.

The model presented here is very practical and easy to use for professionals from the various healthcare services. It can be used with both children and adults, with or without verbal communication. Through the information observed in the Sensory Assessment, it allows us to draw up a Sensory Profile of the person, in order to have a baseline that indicates to care professionals the objectives to be considered in the planning of day to day care for the person, and of specific interventions in the Snoezelen Room that are as targeted as possible.

\section{Sensory profile}

Before beginning a Snoezelen intervention with a patient, it is essential to have previously established hi/her Sensory Profile. Professionals require a framework that provides a baseline from which to plan an intervention in the most appropriate way. The Sensory Profile should be an instrument that is simple to use for professionals who work in the care of people in vulnerable conditions (dementia, Alzheimer's, intellectual disability, autism spectrum disorders, etc.). It should be a tool that can be used in the workplace, in specialized institutions, in schools, etc. Finally, it should be a practical tool that does not require apparatus and instruments unavailable to these professionals.

We will now present a tool designed for this purpose, taking into account the fact that it is a tool with limited reliability, and involves a certain degree of subjectivity on the part of the professional who applies it. A totally reliable way to perform a sensory profile could be, for example, from Evoked Potentials. That is, to present a certain sensory stimulus to a person and to record the brain responses to the given stimulus with a polygraph machine. We know that this way of proceeding would be more reliable and error-free. However, we also know that a methodology of this type is not applicable in most institutions and services that conduct Snoezelen interventions. These institutions do not have polygraph instruments or trained professionals able to use and interpret them. Thus, we need a tool that allows us to establish a Sensory Profile as reliably as possible, albeit with a certain margin of error. It should also be noted that in the group of professionals who care for people with types of vulnerability such as those mentioned above, there has not been a tradition of measuring and evaluating people's sensory capacities as objectively as possible. Nevertheless, we think that having a tool like the one we propose here will be of great help to these professionals.

The Sensory Profile that we propose has been designed according to two basic premises: it should be easy to use (the professionals who perform the Snoezelen intervention are 
familiar with the tool; thus it should not cause any difficulties); and it should be quick to use (it does not require excessive time to complete it, since professionals are often short of time). In addition, it should be able to generate as much information as possible about the person's sensoriality, so that we can design Snoezelen interventions in an appropriate and specific way according to the individual characteristics of the person.

We will refer first to the Sensory Assessment, i.e., to the results recorded and observed when we offer the person different magnitudes and types of sensory stimuli. From the observations we register the types of response of the person, of liking or rejection, to each sensory sensation that we present. When the Sensory Assessment has been completed, the Sensory Profile can be prepared: a record of the most significant results for each sense. The Sensory Profile will generate the baseline from which we can plan interventions with the person in the most appropriate way, either in terms of activities of daily life and activities in general, and, more specifically, in the design of sessions in the Snoezelen Room.

\section{Sensory Assessment}

\section{The context to perform the Sensory Assessment (Methodology Guide)}

The Sensory Assessment of a person will take place on a specific day, in a specific context. We should be aware that on this day, the person will be in a certain condition, as will the professional who performs the assessment. In light of this, we should bear in mind that the results of the Sensory Assessment are to be taken as a guide, providing basic information that may be subject to a degree of bias. However, it will, nevertheless, be a starting point that can mark the beginning of the multisensory intervention with the person.

Place: it is recommended that the sensory assessment be conducted in a quiet room which is not subject to distractions that may affect the results of the Assessment. We want to assess the person's responses to the stimuli that we present to them rather than their responses to unintended environmental input that may also affect the results. A Snoezelen Room is a good place to perform the Sensory Assessment, since it is a quiet, isolated place, in which environmental stimuli can be controlled. If a Snoezelen Room is not available, it is recommended that a space be found that is as quiet and isolated as possible, such as the person's own room, a suitable room in the facility, an office, etc. Position and predisposition of the person: when starting the Sensory Assessment, it is essential to guarantee the well-being and comfort of the person. We want to assess his/her responses to the stimuli that we will present to him/her, and not his/her response to general discomfort or distress, for example. Therefore, it is necessary to position the person comfortably, making allowances for their condition, postural control, and preferences. We should also ensure that he/she is comfortable in terms of clothing and other external factors that may influence his/her comfort. 
Professionals and registration: it is recommended that two professionals be present during the Sensory Assessment. One should present the different stimuli to the person. Since the person should be made to feel as safe as possible, it will facilitate the process if the professional is known to the patient. If not, it is necessary to devote time to building trust. The second professional is the observer, who will record the person's responses to the different contexts of the stimulus. This record may be visual (a recording of the assessment session) and/or written (a recording of the person's responses in writing on an assessment sheet), and ideally both should be made. Recording the sensory evaluation will allow us to observe behavioural responses that perhaps may have gone unnoticed, revealing sensory sensations that are important for the emotional well-being of the person.

Materials: we should have the materials necessary to carry out the sensory assessment to hand. Materials should be specially selected and should not require overly technical elements.

Sensory Assessment Methodology: in practice we work with Sensory Assessment sheets (created as part of the methodology for evaluating the person's sensory responses in different situations).

Behavioural responses that we assess: the Sensory Assessment sheets are designed to record responses of people with high levels of dependency and/or with difficulties in verbal communication. It is important to note that answers can be adapted to take the user's profile into consideration. They are qualitative, observational responses through which we try to record, above all, the positive or negative reactions elicited by each sensory stimulus. The responses recorded are:

- Eye movements: record whether the person makes eye movements in response to the presented stimulus.

- Avoidance/withdrawal: record whether the person shows avoidance behaviours in response to the presented stimulus.

- Vocalizations/verbalizations of pleasure or displeasure: record whether the person demonstrates non-verbal or verbal expressions of pleasure or displeasure in response to the presented stimulus.

- Voluntary movements/gestures: assess whether the presented stimulus generates voluntary movements in the person.

- Modification of muscle tone: record whether the presented stimulus generates variation in muscle tone in the person. Variation can be in the sense of relaxation (decreased muscle tone) or activation (increased muscle tone).

It should be noted that in addition to the above-mentioned responses, it is also important to note down and qualitatively record any other responses from the person which we consider significant and which is not included in the previous sections.

We will now look at how the sensory assessment is conducted for each sense.

Visual Sensory Assessment: three micro-capacities will be assessed in the visual sensory response:

- Visual reaction: to assess whether the person reacts when a visual stimulus is presented in different magnitudes and experimental situations. 
- Visual fixation: to assess whether the person, in addition to his/her reaction, is able to fix his/her gaze on the visual stimulus presented.

- Visual monitoring: in order to assess whether the person is able to follow the visual stimulus presented, the visual stimulus is moved gradually, first in a cross pattern (from top to bottom and from left to right), then diagonally, and finally, in a circle. We observe if the person follows the visual element presented with his gaze as we move it.

The assessment is conducted in three environmental situations:

- Maximum light or normal ambient light: with lights or with natural daylight.

- Dim light: it is important to have a dimmer switch for ambient light to be able to slightly reduce the intensity of the light.

- Darkness: the room is left in darkness, without light. A light stimulus is presented and the responses are observed. This environmental condition is especially indicated for those with poor visual acuity. The contrast between light and dark means we can see whether there is any type of reaction.

The stimuli that are presented in the visual assessment are:

- Personal stimulus: the professional him/herself is presented as the stimulus. We observe how the user reacts when he/she sees the caregiver.

- Attractive visual stimulus: an attractive visual stimulus with striking colours is presented to the person.

- Luminous stimulus: A luminous element (a bundle of optical fibres, a flashlight, or any element that emits light) is presented, typically in the dark, but it can also be shown in the other environmental conditions

Sensory Auditory Assessment: two micro-capacities will be assessed in the responses to auditory sensory stimuli:

- Auditory reaction: to assess whether the person shows any type of reaction when presented with an auditory stimulus in different magnitudes and experimental situations.

- Auditory localization: to assess whether when presented with an auditory stimulus in different magnitudes and different experimental situations, the person tries to locate the origin of the sound source with head, eye, or body movements.

The assessment will be carried out with three different intensities of magnitude of auditory stimulus:

- Low intensity of auditory stimulus: a quiet sound is emitted.

- Intermediate intensity of auditory stimulus: a medium sound is emitted (the sound intensity is neither low nor high).

- High intensity of auditory stimulus: a loud sound is emitted.

The types of auditory stimuli that we will present are:

- The Human Voice: the professional who carries out the assessment addresses the person, saying his/her name in the three aforementioned intensities (low, intermediate, and strong), for several repetitions at each intensity. 
- High-pitched sound: the person will be presented with an auditory stimulus with a high-pitched sound, such as a musical instrument, e.g., a triangle. There will be several repetitions.

- Low-pitched sound: the person will be presented with a sensory stimulus of a lowpitched nature, such as a Tibetan bowl, a drum, etc. There will be several repetitions.

- Vibration: a vibratory sensation will be presented to the person using an element that generates this sensation, such as an electric toothbrush or any other object that vibrates. Several repetitions will be carried out.

Tactile Sensory Assessment: in this assessment, it is important that the person feels comfortable and safe, to avoid causing them discomfort. This assessment should be performed directly on the skin, on the upper extremities (arms and hands) and on the lower extremities (legs and feet), provided that the person accepts it. In some cases, if the person accepts it readily, we might assess their reaction to tactile stimulus on the face and head and on any other part of the body, such as the trunk; however, normally only the upper and lower extremities are assessed, since the person feels more comfortable with this and the procedure is less invasive. For the extremities we differentiate between the two hemibodies (right and left). This information is significant for some patients, since, due to certain neurological disorders, they might present different tactile sensitivity on one half of the body and the other.

The tactile stimuli that we present are: temperature, texture, and pain.

- Warm stimulus: a stimulus that warms the person but not excessively. For example, a material that can be heated in the microwave such as seeds placed in a sachet or other objects that generates heat, such as a hair dryer.

- Cold stimulus: a stimulus that is cold, such as ice cubes wrapped in a cloth.

- Soft stimulus: a stimulus with a soft texture, such as a soft fabric, a sponge, cotton wool etc.

- Rough stimulus: a stimulus that is rough or coarse, such as a scouring pad, a strong bristle brush, a rope mitt etc.

- Sharp stimulus: a sharp instrument, such as a needle, pricks different points on the person's skin.

We therefore observe the responses of the person to varied temperatures, varied textures, and pressures on different parts of the skin.

Proprioceptive Sensory Assessment: the good positioning or comfort of the person is important in this assessment. The person might be stretched out on a bed, in a comfortable chair, on a mat, etc. It is important to adapt to his/her postural control, and to try to perform the assessment as reliably as possible.

We assess proprioception in the upper and lower extremities. If the person accepts it, we can also try to apply pressure to the head and trunk; however, often, the head and trunk are too invasive, and we limit ourselves to the extremities. We assess both hemibodies. In this sense, it is important to observe if there are responses of pleasure or rejection 
depending on laterality. We are interested in assessing whether the person feels pain in certain parts of their body.

The proprioceptive assessment is performed by applying firm pressure on each limb at a slow pace and repeating the procedure on each limb between three and five times. The sequence is:

Upper limbs:

- Shoulder, arm, elbow, forearm, wrist, and hand.

Lower limbs:

- Hip, thigh, knee, leg, ankle, and foot.

We repeat the procedure at a slow pace, applying firm pressure in the aforementioned sequences, repeating them at least five times, and always looking out for responses of acceptance or rejection from the person. If he/she has verbal communication, we also ask him/her directly whether the stimulus is pleasant or unpleasant.

Olfactory and gustatory assessment: the assessment of preferences and capacities for olfactory and gustatory sensory discrimination is essential in our day-to-day life. To assess this sense, varied and contrasting sensory sensations in terms of smell and taste are provided. We try to observe which sensations the person prefers, which sensations he/ she dislikes, and we observe, in particular, whether he/she can identify what should be unpleasant stimuli so that in everyday situations, he/she is able to discern harmful stimuli or those that might affect their well-being.

Olfactory sensory evaluation stimuli: we present various and contrasting olfactory stimuli to the person and observe his/her responses.

Possible olfactory stimuli to present:

- Floral, spicy, acidic, sweet, unpleasant smells.

Taste sensory evaluation stimuli: we will present different flavours to be tasted by the person. The aim is to present various contrasting flavours in order to observe the responses of the person to different sensations, and to record their taste preferences and dislikes.

Possible taste stimuli to present:

- Salt, sugar, bitter taste, sour taste etc.

Vestibular sensory evaluation: assessing the responses of acceptance or rejection of the person when presented with various vestibular sensory sensations is important in order to offer him/her the most appropriate care and day-to-day activities for their well-being. In this sense, it is necessary to apply the vestibular inputs gradually, always observing the verbal and, above all, non-verbal responses, such as nystagmus (rapid and involuntary movements of the eyes that can be: from one side to another (Horizontal nystagmus); up and down (vertical nystagmus); or rotating (rotational or torsional nystagmus, skin coloration, etc).

The vestibular assessment can be carried out in different contexts, depending on the materials available. If we have one, we can perform the assessment in a vestibulator. If not, we can do it on a swing. With adults, if we do not have the aforementioned elements, it can be performed in a wheelchair, in a crane, on a seesaw, or in an office chair with wheels. 
The aim is to present various vestibular movements and to observe the person's responses to them. It is very important to proceed cautiously, always looking at the person's eyes and face for signs of discomfort or overstimulation.

Experimental situations to assess the person's response to vestibular sensations:

- Response to linear acceleration: five swings in the frontal plane are performed, followed by five swings in the sagittal plane.

- Response to angular acceleration: five clockwise turns are made, followed by five anticlockwise turns.

- Responses to vertical acceleration: five vertical jump movements are performed, for example, on a raised mat.

- Response to inversion: the patient is turned upside down three-five times.

The final two situations (vertical acceleration and inversion) are only carried out with children.

Once the Sensory Assessment has been carried out, a Sensory Profile is prepared. This document, explained in section 2, summarizes the most significant results in terms of the person's positive and/or negative response when exposed to each of the sensory sensations, and a description of the response that the person emits. The Sensory Profile, therefore, is a document in which we synthesize the person's preferences or dislikes and describe his/her responses to them. From this document, the sensory objectives on which to work with the person can be established. These objectives involve recommendations for daily life that should provide a more pleasant daily life with greater emotional well-being for the person. In addition, they will serve as a guideline for multisensory activities appropriate to the person and, especially, they will indicate the objectives to be developed in sessions in the Snoezelen room.

\section{Examples of the repercussion of sensory profile results on everyday life}

In this section we will provide examples of Sensory Assessments and Profiles, and how they might generate 24-hour Snoezelen objectives and proposals for interventions in the Snoezelen Room. The improvement in Quality of Life of the person and in their Emotional Well-being is clearly demonstrated. Only the most important results that generated a significant change are included, rather than verbatim sensory assessments.

\section{Older person with cognitive impairment}

A 100-year-old lady with a level II Dependency, who lives at home with home support and has significant health problems in terms of low weight (37 kilos) and a refusal to take protein and vitamin supplements recommended by her doctor (due to the fact that she is a naturopath and only ingests natural foods, without additives or preservatives). In addition, she has behavioural problems in that she rejects clothes that the home caregivers offer her. 
- Visual sensory evaluation: she prefers bright colours, declaring that she hates dark colours such as brown and grey. Based on this assessment, the colours of her clothes were changed. She was offered clothing in more brighter colours, which led to the end of behavioural difficulties when dressing her.

- Olfactory sensory evaluation: she likes the smell of vanilla. As a result, the social worker bought a natural vitamin supplement with a vanilla aroma from the pharmacy. She took it willingly and in a few weeks gained three kilos in weight, improving her overall health.

\section{0-year-old girl with Autism Spectrum Disorder}

A person with ASD and severe intellectual disability, without verbal language and with behavioural problems (rejection, yelling, and hetero-aggression) at school and in daily activities at home, such as showering.

- Touch sensory evaluation: she has a preference for cold temperatures, showing a clear rejection of warmer temperatures. She demonstrates a preference for coarse textures over soft textures. Based on this assessment, it was recommended that the family shower her with cold water, using a rough sponge. Subsequently, rejection behaviours in the shower were clearly reduced.

- Auditory sensory evaluation: rejection responses to high intensity sounds were clearly observed in the sensory evaluation, regarding both the human voice and lowand high-pitched sounds. In classroom situations with loud noise or disturbance from other classmates involving shouting or a high tone of voice, the teacher was advised to invite the girl to another calmer classroom space. In this way, behavioural disturbances in the school classroom were reduced.

\section{Challenges and conclusions}

The Sensory Profile presented here should be regarded as a tool for qualitative observation, available to professionals who require clear and reliable knowledge of the responses of children and adults for whom they should plan and carry out an intervention on a sensory basis. Mention has been made of the possible bias that may be implicit in the presented tool, since it is an observational instrument. The evidence from the many professionals from different caring fields (teachers, occupational therapists, psychologists, physiotherapists, etc.) who have used the Sensory Profile and Sensory Assessment is that it results in a better knowledge of the patient, and above all, a better understanding of his/her behaviour and emotions.

It is important that there be two professionals when performing the Sensory Assessment. As mentioned above, one presents the stimuli, and is in direct contact with the person, while the other has a more discreet role as the observer who records the responses of the person to different sensations. It is important after the sensory evaluation that both professionals analyse and review the results so that a sensory profile can be created 
with the highest possible reliability, in order to offer intervention objectives appropriate to the needs and abilities of the person; thereby improving relationships, activities of daily living, and activity scheduling.

The observational tool presented in this article can be a good complement to other standardized tests such as Dunn's Sensory Profile. On the one hand, the structured observation presented in this article allows us to collect responses in an "experimental situation", whereas Dunn's sensory profile collects information from parents and teachers in everyday contexts. In both cases, we start from an ecological model and positive psychology, taking account of the environment and strengths (in terms of the abilities and preferences) of the person.

It is intended to provide professionals with a means to a deeper understanding of the people to whom they should direct their attention and care. We regard this tool as a starting document used by many professionals with concrete results in interventions that have resulted in clear improvements in the day-to-day life of patients in general terms and in terms of the design of sessions in the Snoezelen Room.

\section{References}

Brown, C. et al. (2001). The Adult Sensory Profile: Measuring Patterns of Sensory Processing. The American Journal of Occupational Therapy, 55(1), 75-82.

Dunn, W. (1994). Performance of typical children on the Sensory Profile: An item analysis. American Journal of Occupational Therapy, 48, 967-974.

Dunn, W. \& Westman, K. (1997), The Sensory Profile: The performance of a National Sample of children without disabilities. The American Journal of Occupational Therapy, 55(1), 25-34.

Tomchek, S. \& Dunn, W. (2007), Sensory Processing in Children With and Without Autism: A comparative Study using the Short Sensory Profile, 61(2), 190-200.

Williams, S. W. \& Shellenberger, S. (1996) How does your engine run? A leaders guide to the alert program for self-regulation. Albuquerque, TherapyWorks, Inc. 651 Strategies for sensory processing disorders. 


\section{Authors}

Cid Rodriguez, Maria José, Ph.D.

Psychologist, coordinator ISNA Spain, collaborator University of Lleida.

C/Major, n53. CP43570. Santa Bàrbara. Tarragona. Spain

mariajosecidrodriguez1964@gmail.com

Ribes Castells, Ramona, Ph.D.

Professor in the department of psychology. Faculty of education, psychology and social work. University of Lleida.

avd. Estudi General, n4. CP.25180. Lleida. Spain

ramona.ribes@udl.cat

Mgr. Janků, Kateřina, Ph.D.

Assistant Professor at the Institute of Special Education, Faculty of Public Policy, Silesian University in Opava

Bezručovo náměstí 885/14, 74601 Opava, Czech Republic

katerina.janku@fvp.slu.cz 